\title{
BMJ Open The benefits of hardware removal in patients with pain or discomfort after fracture healing of the ankle: a systematic review protocol
}

\author{
Alexandra Thune, Mårten Hagelberg, Hans Nåsell, Olof Sköldenberg
}

To cite: Thune A, Hagelberg M, Nåsell $\mathrm{H}$, et al. The benefits of hardware removal in patients with pain or discomfort after fracture healing of the ankle: a systematic review protocol. BMJ Open 2017;7:e014560. doi:10.1136/ bmjopen-2016-014560

- Prepublication history and additional material for this paper are available online. To view these files please visit the journal online (http://dx.doi. org/10.1136/bmjopen-2016014560).

Received 5 January 2017 Revised 21 June 2017 Accepted 30 June 2017

CrossMark

Department of Clinical Sciences, Karolinska Institutet, Danderyd Hospital, Danderyd, Stockholm, Sweden

Correspondence to Dr Olof Sköldenberg; olof.skoldenberg@ds.se

\section{ABSTRACT}

Introduction For any orthopaedic surgeon working with trauma; ankle fractures are one of the most common injuries treated. The treatment of ankle fractures can be conservative, using external fixation, but more commonly the fractures are treated with open reduction and internal fixation. Residual pain and discomfort are common in patients after surgical treatment of fractures of the ankle. Sometimes it is difficult to determine whether the pain or discomfort is due to the implants left in situ or the primary injury itself. In many cases, the decision is made to remove the implants. Extraction of internal fixation material from the ankle is a common procedure in many orthopaedic clinics. There are no evidence-based guidelines or consensus regarding the effect of hardware removal from the ankle. The aim of this protocol is to describe the method that will be used to collect, describe and analyse the current evidence regarding hardware removal after fracture healing of the ankle.

Methods and analysis We will conduct a systematic review of studies that were published after 1967 regarding the benefits of hardware removal in patients with pain or discomfort after fracture healing of the ankle. Study selection will follow the Preferred Reporting Items for Systematic Reviews and Meta-Analysis guidelines. We will make a predefined search strategy and use it in several databases. We will include both randomised controlled trials (RCTs) and non-RCT studies. We will use descriptive statistics to summarise the studies collected. If more than one RCT is collected then a meta-analysis will be conducted. The quality of evidence will be assessed using Grading of Recommendations Assessment, Development and Evaluation guidelines.

Ethics and dissemination No ethics approval is required as no primary data will be collected. Once complete, the results will be made available by peer-reviewed publication.

Trial registration number PROSPERO registration number CRD42016039186

\section{BACKGROUND}

\section{Rationale}

Ankle fractures are among the most common fractures and are often treated with surgery using metal implants. ${ }^{1}$ The internal fixation often consist of some kind of metallic implant

\section{Strengths and limitations}

- Hardware removal of the ankle after fracture healing is a very common procedure and this study will be relevant to many orthopaedic clinics and patients.

- We hope to provide evidence-based data on outcome following hardware removal from the ankle after fracture healing in adults.

- Studies published in other languages than English may lead to language bias and are therefore excluded. However, this may also lead to exclusion bias as relevant studies may be published in other languages.

- Randomised controlled trials with comparable outcomes may not be available for meta-analysis.

such as screws, plates and cerclage. After fracture healing, the implants are then either removed or left in situ. The incidence of ankle fracture peaks in elderly women due to osteoporosis and in younger men related to high-energy trauma. ${ }^{2}$ Ankle fractures account for $9 \%$ of all adult fractures. ${ }^{3}$ The majority of patients surgically treated for ankle fractures report high rates of functional outcomes, ${ }^{45}$ and in a long-term follow-up, this seems to improve even further. ${ }^{6}$ However, persistent pain is common among patients surgically treated for ankle fractures. ${ }^{7}$ Once the fracture has healed, the implant has no further function. There are different opinions among surgeons as to whether the metal implants should be routinely removed after fracture healing or not. The indication to remove the hardware is often pain relief and improved function of the ankle but the procedure can also be associated with complications such as infection, neurovascular damage or even refractures. A survey from 2008, where surgeons from 65 countries took part, showed that $58 \%$ of the participants did not agree that routine implant removal was necessary in general (not limited to ankle fractures) and 
$85 \%$ of all the participants concurred that implant removal poses a burden to hospital resources. ${ }^{8}$ In 1996, a study from Finland showed that of all orthopaedic operations in Finland 6.3\% were regarding removal of implants, with implants of the ankle being the most common. ${ }^{9}$ There are currently no evidence-based guidelines on removal of implants after healing of ankle fractures. A systematic review is needed to assess the evidence available and to determine if further studies are required.

\section{Objectives}

The objective of this study is to systematically review the literature for qualitative evidence that explores patient outcomes regarding pain and patient satisfaction in adults after implant removal following fracture healing of the ankle.

\section{METHOD AND ANALYSIS}

This protocol will conform to the Preferred Reporting Items for Systematic Review and Meta-Analysis Protocol (PRISMA-P) guidelines. ${ }^{10}$

\section{Eligibility criteria}

This protocol is developed on the basis of population, intervention, comparators and outcomes questions.

\section{Population}

We will include studies examining all human adults $(\geq 18$ years) surgically treated for any type of traumatic fracture of the ankle.

\section{Intervention}

Intervention of interest is hardware removal from the ankle after fracture healing. Removal of syndesmotic screws is not included as this is a common routine treatment in many orthopaedic institutions that aims to increase joint movement rather than pain relief. ${ }^{11}$ Type of hardware (plates, screws, material, model or manufacturer) will not be differentiated.

\section{Comparison}

For the intervention, all comparators will be of interest. All studies except systematic reviews and case studies will be included in the systematic review.

\section{Outcome}

Reduced pain and patient satisfaction are of primary interest. If reported on, this will be analysed and graded. Pain will be assessed using the visual analogue scale $(0-100)$ or any other analogue pain scale. Patient satisfaction will be assessed using any self-reporting form. Self-reporting forms anticipated to be used include 36-Item Short-Form Health Survey (SF-36), ${ }^{12}$ Short-Form McGill Pain Questionnaire ${ }^{13}$ and Short Musculoskeletal Functional Assessment. ${ }^{14}$ The scores will be modified to make comparison possible. A standardised mean difference will be calculated for each study and all scales will be modified so that lower score is the worse outcome.
Secondary outcomes will be complication rate of wound infections following intervention or refracture within the time of follow-up. The complication rate will be measured as the percentage of included patients in the studies.

\section{Search strategy}

The search strategy will be constructed by the first author. A librarian with expertise in healthcare databases and systematic reviews will be consulted. Literature search will be conducted using medical subject headings and text words related to hardware removal after fracture healing of the ankle. Searches will be done in PubMed/ MEDLINE, Cochrane Collaboration and Embase. Only studies written in English will be included. Because of the constant change and development of surgical technique and implants, we decided to limit our search to the past 50 years. Studies published before 1967 will be excluded. The reference list of eligible studies will be scanned. Should the time consumption of the review process be $>12$ months, an updated search will be made to include all the latest articles that might be of interest. The initial search strategy in PubMed is presented in an online Supplementary file 1.

\section{Study records}

Search results from PubMed/MEDLINE, Cochrane Collaboration and Embase will be downloaded and managed in Endnote X7 (Thomson Reuters, Philadelphia, Pennsylvania, USA). Two members of the review team (AT and $\mathrm{MH}$ ) will independently screen the titles and abstracts yielded by the search against the inclusion criteria. The selected studies will then be studied in full text by AT and MH who will assess if they meet the inclusion criteria. If there is disagreement whether a study meets the inclusion criteria or not, a third party (OS) will make the final decision. Rejected articles in the search will be commented on and filed for record. Neither of the review authors will be blind to the journal titles or to the study authors or institutions. A standardised data collection form, Research Electronic Data Capture, ${ }^{15}$ will be used to extract data that includes all patient-related outcomes. Data extraction will be carried out by AT and then verified by $\mathrm{MH}$ to reduce bias. If disagreement or discussion occurs, a senior member of the review team (OS) will act as an arbitrator. Data to be extracted are publication year, author, study design, size of population, time to follow-up, drop-out rate, patient-reported outcome score(s), complication rate, mean age and gender. All authors will read and have the chance to contribute to the final report.

\section{Risk of bias assessment}

For randomised controlled trial (RCT) studies, the Cochrane Collaboration tool for assessing the risk of bias will be used. ${ }^{16}$ The methodological quality of included non-RCT studies will be evaluated using the validated Newcastle-Ottawa scale (NOS) ${ }^{17}$ as recommended by the Cochrane Non-Randomized Studies Methods Working 
Group. Using NOS, the quality of a study will be judged on the selection of the study groups, the comparability of the groups and the ascertainment of outcome of interest. Each study included will be investigated and judged by AT and MH independently. In case of discrepancy or disagreement, OS will act as an arbitrator. Studies with high risk of bias will be omitted.

\section{Data synthesis and analysis}

In the event of the search strategy yielding two or more comparable randomised studies, a meta-analysis will be conducted.

For non-RCT studies, we will use descriptive statistics to summarise characteristics and findings of the included studies.

The quality of evidence for all outcomes in the included studies will be graded using the Grading of Recommendations, Assessment, Development and Evaluation (GRADE) working group methodology. ${ }^{18}$ The GRADE score is based on study quality, inconsistency of result, indirectness of evidence, imprecision, publication bias, large magnitude of effect and effect of plausible residual confounding. The final GRADE score is divided into four categories (high, moderate, low or very low) reflecting the quality of the evidence.

If important data are missing attempt will be made to contact the corresponding author.

If we need to amend this protocol, we will give the date of each amendment, describe the change and give the rationale. Changes will not be incorporated into the protocol.

\section{DISCUSSION}

Today, removal of metal implants after ankle surgery is a common surgical procedure in many orthopaedic clinics. Whether this procedure is beneficial to the patient or not is unclear and a systematic review is needed.

A brief scan of the literature prior to this review did not reveal any relevant RCTs and it is possible that this systematic review will therefore not be sufficient to offer evidence-based recommendations on whether or not metal implants should be removed after ankle surgery. In that case, the systematic review may reveal that further studies are needed.

\section{Acknowledgements The authors thank librarian Alena Haarmaan for help in} constructing the database search strategy.

Contributors AT: the main author of the protocol and wrote the final report. AT and MH: selected studies and did data extraction. OS: acted as an arbitrator in case of disagreement and supervised AT and MH. HN: an advisor for AT throughout the process. All authors: read and provided input for improvements of the final report.

Funding This research received no specific grant from any funding agency in the public, commercial or not-for profit sectors.
Competing interests None declared.

Patient consent No patient consent needed, systematic review.

Provenance and peer review Not commissioned; externally peer reviewed.

Data sharing statement No additional data available.

Open Access This is an Open Access article distributed in accordance with the Creative Commons Attribution Non Commercial (CC BY-NC 4.0) license, which permits others to distribute, remix, adapt, build upon this work non-commercially, and license their derivative works on different terms, provided the original work is properly cited and the use is non-commercial. See: http://creativecommons.org/ licenses/by-nc/4.0/

(c) Article author(s) (or their employer(s) unless otherwise stated in the text of the article) 2017. All rights reserved. No commercial use is permitted unless otherwise expressly granted.

\section{REFERENCES}

1. Thur CK, Edgren G, Jansson KÅ, et al. Epidemiology of adult ankle fractures in Sweden between 1987 and 2004: a population-based study of 91,410 swedish inpatients. Acta Orthop 2012;83:276-81.

2. Court-Brown CM, McBirnie J, Wilson G, et al. Adult ankle fractures-an increasing problem? Acta Orthop Scand 1998;69:43-7.

3. Court-Brown CM, Caesar B. Epidemiology of adult fractures: a review. Injury 2006;37:691-7.

4. Shah $\mathrm{NH}$, Sundaram RO, Velusamy A, et al. Five-year functional outcome analysis of ankle fracture fixation. Injury 2007;38:1308-12.

5. Lindsjö U. Operative treatment of ankle fracture-dislocations. A follow-up study of 306/321 consecutive cases. Clin Orthop Relat Res 1985;199:28-38.

6. Regan DK, Gould S, Manoli A, et al. Outcomes over a decade after surgery for unstable ankle fracture:functional recovery seen 1 year postoperatively does not decay with time. J Orthop Trauma 2016;30:e236-41.

7. Friesgaard KD, Gromov K, Knudsen LF, et al. Persistent pain is common 1 year after ankle and wrist fracture surgery: a registerbased questionnaire study. Br J Anaesth 2016;116:655-61.

8. Hanson B, van der Werken C, Stengel D, et al. Surgeons' beliefs and perceptions about removal of orthopaedic implants. BMC Musculoskelet Disord 2008;9:73.

9. Böstman $O$, Pihlajamäki H. Routine implant removal after fracture surgery: a potentially reducible consumer of hospital resources in trauma units. J Trauma 1996;41:846-9.

10. Stewart LA, Clarke M, Rovers M, et al. PRISMA-IPD Development Group. Preferred Reporting items for systematic review and MetaAnalyses of individual participant data: the PRISMA-IPD statement. JAMA 2015;313:1657-65.

11. Schepers T. To retain or remove the syndesmotic screw: a review of literature. Arch Orthop Trauma Surg 2011;131:879-83.

12. Ware JE, Sherbourne CD. The MOS 36-item short-form health survey (SF-36). I. Conceptual framework and item selection. Med Care 1992;30:473-83.

13. Melzack R. The short-form McGill Pain Questionnaire. Pain 1987;30:191-7.

14. Swiontkowski MF, Engelberg R, Martin DP, et al. Short musculoskeletal function assessment questionnaire: validity, reliability, and responsiveness. J Bone Joint Surg Am 1999;81:1245-60.

15. Harris PA, Taylor R, Thielke R, et al. Research electronic data capture (REDCap)--a metadata-driven methodology and workflow process for providing translational research informatics support. J Biomed Inform 2009;42:377-81.

16. Ghosh A, Robbins K, Kelly J. The Cochrane Library: a resource for current reviews of clinical evidence. Minn Med 2000;83:43-5.

17. Oremus M, Oremus C, Hall GB, et al. ECT \& Cognition Systematic Review Team. Inter-rater and test-retest reliability of quality assessments by novice student raters using the Jadad and Newcastle-Ottawa Scales. BMJ Open 2012;2:e001368.

18. Atkins D, Best D, Briss PA, et al. GRADE Working Group. Grading quality of evidence and strength of recommendations. BMJ 2004;328:1490. 